\title{
Award-winning papers in 2016
}

Papers published in Energy are eligible for awards from the Institution of Civil Engineers. Papers from any of the ICE journals can be nominated for several awards. In addition, each journal has awards dedicated to their specific subject area.

On Friday 6 October 2017, ICE president Tim Broyd presented awards to the following papers published in Energy in 2016. The editorial panel nominated their best papers and an awards committee chaired by Nigel Wright allocated the awards.

\section{James Watt Medal}

The James Watt Medal, for papers with substantial mechanical engineering content, was awarded to Klumpp (2016).

\section{Abstract}

In this paper, technologies are analysed that exhibit potential for mechanical and chemical energy storage on a grid scale. Those considered here are pumped storage hydropower plants, compressed air energy storage and hydrogen storage facilities. These are assessed and compared under economic criteria to answer the question of which technology is to be favoured. For this purpose, the levelised electricity cost for various dispatch scenarios - short-, medium- and long-term storage - are calculated for the present and for 2030. Fundamental indicators considered are their respective efficiencies, capital expenditure and operational expenditure, and technical service lives. From an economic point of view, today pumped hydro is the most cost-efficient short- and medium-term storage technology, closely followed by compressed air energy storage. In the future, too, there will be no fundamental change in this result, even with optimistic assumptions for the development of hydrogen storage. However, hydrogen storage is becoming more competitive and represents the most economic option in the future for long-term energy storage.
\end{abstract}

\section{David Hislop Award}

The David Hislop Award, presented to the best paper published on heavy marine design and construction with particular reference to offshore engineering, was awarded to Binnie (2016).

\section{Abstract}

The Severn estuary in Britain has the second highest tidal range in the world. However, the estuary and much of the adjoining Bristol Channel are too shallow for current commercial tidal stream arrays of the horizontal-axis type. The most successful tidal range scheme is the La Rance scheme in Brittany, France, which has operated for 45 years and produces the cheapest electricity in Europe. Tidal range schemes in the Severn estuary have been studied several times. The Department of Energy and Climate Change study in 2008-2010 showed a Cardiff/Weston ebb-only barrage (15.6 TWh/year) and the Bridgwater Bay ebb/flood lagoon (6.2 TWh/year) to be feasible, but with appreciable environmental issues. More detailed environmental and planning studies would be needed to demonstrate compliance with the EU Habitats Directive. Several smaller lagoon schemes have also been proposed and the Swansea Bay scheme is $0.4 \mathrm{TWh} /$ year currently seeking planning permission. Thus, the total energy output from tidal range in the Severn could be about $25 \mathrm{TWh} /$ year, about $7 \%$ of the

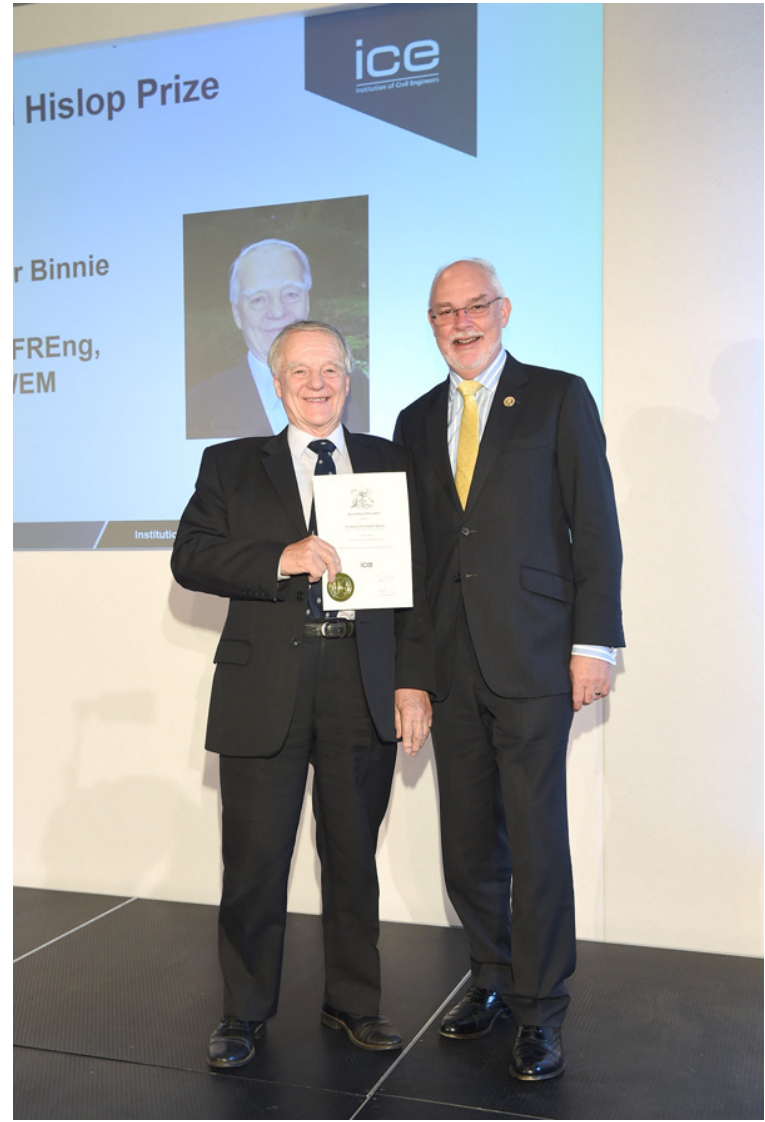

Chris Binnie, winner of the David Hislop Award, with ICE president Tim Broyd

UK energy needs. The energy available would be predictable and, if aligned with tidal lagoons on the North Wales coast and elsewhere, continuous, although varying with the spring and neap tidal cycles.

\section{REFERENCES}

Binnie C (2016) Tidal energy from the Severn estuary, UK. Proceedings of the Institution of Civil Engineers - Energy 169(1): 3-17, https://doi.org/10.1680/jener.14.00025.

Klumpp F (2016) Comparison of large-scale energy storage technologies. Proceedings of the Institution of Civil Engineers Energy 169(4): 148-160, https://doi.org/10.1680/jener.14.00043. 